\title{
Le torus palatin. Etude réalisée sur 723 crânes humains et comparaison avec les données de la littérature
}

\author{
Torus palatinus: data collected from 723 human skulls compared to data \\ reported in the literature
}

JEAN HISS ${ }^{\star}$, CORINNE TADDEI ${ }^{\star *}$, RENEE WOLFRAM-GABEL ${ }^{* \star *}$, AHMED FEKI ${ }^{\star}$

\begin{abstract}
RÉSUMÉ
Le torus palatin est une « tumeur bénigne » constituant une élévation de la ligne médiane du palais. Décrite en 1814, cette particularité anatomique est actuellement classée selon quatre types: torus plat, fusiforme, nodulaire et lobulaire. Cette exostose du maxillaire, rare avant l'âge de dix ans, croît jusqu'à l'âge de 30 ou 40 ans pour atteindre un plateau, généralement suivi par une légère phase de décroissance. L'apparition du torus palatin résulterait de l'action combinée de facteurs génétiques et environnementaux. Selon cette théorie, le franchissement d'un seuil se révèle nécessaire pour que ces deux facteurs conduisent à la formation d'un torus.

La fréquence du torus palatin est variable selon les études. Une étude basée sur l'observation de 723 crânes, d'origines ethniques diverses, appartenant à la collection d'ostéologie de l'Institut d'Anatomie Normale de Strasbourg, donne des résultats similaires aux données de la littérature. Une fréquence relativement importante du torus palatin est cependant retrouvée dans la population d'origine rhénane. (Med Buccale Chir Buccale 2005; 11 : 205-213).
\end{abstract}

mots clés : torus palatin, étude épidémiologique, anatomie

médecine buccale chirurgie buccale

VOL. $11, \mathrm{~N}^{\circ} 4$ 2005

page 205

\section{SUMMARY}

The torus palatinus is well known as a benign neoplasm building up a swelling on the palatal median line. Described since 1814, this anatomical peculiarity is classified, nowadays, in four types: flat, spindle-shaped, nodular or lobular torus. This maxillary exostosis, seldom seen before the age of ten, increase until the age of thirty or forty while its growth reach a steady level, followed by a decreasing stage. The coming out of torus palatinus would be triggered by genetically factors associated with environmental factors. According to this theory, a threshold crossing is indeed necessary to allow a torus to happen, ted by these factors.

The prevalence of the torus palatinus is variable depending on the reports. This study is based on the examination of 723 human skulls, of various ethnical descents, stored in the collection of osteology at the institute of normal anatomy, Faculty of Medicine of Strasbourg. The examination of this sample show similar results to data of the literature concerning this topic. Nevertheless, a fairly high frequency of torus has been observed on the skulls coming from the population of the middle rhine basin. (Med Buccale Chir Buccale 2005; 11: 205-213).

key words: torus palatinus, epidemiological study, anatomy

\footnotetext{
* Département de Médecine Buccale et de Chirurgie Buccale, Service d’Odontologie, Hôpitaux Universitaires de Strasbourg

** Département de Prothèse Amovible, Service d'Odontologie, Hôpitaux Universitaires de Strasbourg

*** Institut d'Anatomie Normale, Faculté de Médecine de Strasbourg
}

Demande de tirés à part : 
médecine

buccale

chirurgie

buccale

VOL. $11, \mathrm{~N}^{\circ} 4$ 2005

page 206
Le torus palatin est une tumeur osseuse bénigne du maxillaire constituant une élévation de la ligne médiane du palais, sur la suture cruciforme qui unit les os palatins et maxillaires [1].

De nombreuses classifications ont été proposées depuis la première description par Fox en 1814, qui présente le torus palatin comme une exostose détruisant la forme régulière du palais [2].

En 1842, Carabelli, pour qui le torus palatin n'est déjà qu'une variation anatomique du palais, décrit le torus palatin comme une protrusion d'os ayant la forme et la longueur d'un demi œuf de pigeon [3]. Au début du XX' siècle, Ledouble [4] distingue déjà deux formes principales dont le grand diamètre correspond toujours à la suture palatine médiane : une forme étroite ou en fuseau et une forme large ou triangulaire à base postérieure arrondie. Des classifications plus complexes apparaissent rapidement. En 1929, Dorrance [2] isole huit types de torus palatins. Martin, en 1973 propose une méthode de classification basée sur le caractère linéaire ou ovalaire du torus palatin [5,6]. Thoma (1937) rend obsolètes les classifications antérieures. II propose une description à la fois simple et complète, et classe toutes les formes prises par le torus palatin selon quatre types: le torus plat, le torus fusiforme, le torus nodulaire et le torus lobulaire $[7,8]$. Cette classification fait actuellement consensus puisqu'elle permet de regrouper simplement les différentes formes cliniques pouvant être prises par cette exostose [9] (Fig. 1). L'histologie des différents types de torus montre que cette excroissance osseuse est composée d'os spongieux à sa partie centrale, entouré d'un os cortical dense.

Cette exostose du maxillaire peut apparaître durant les stades précoces du développement [10]. Sa présence a été signalée sur des fœtus [11], des nouveau-nés $[1,12,13]$ et des enfants $[1,10,14]$. Le torus palatin est rare avant l'âge de dix ans, et il croît jusqu'à l'âge de trente ou quarante ans pour atteindre un plateau $[8,15,16]$, généralement suivi par une phase légère de décroissance [17]. Cette croissance peut cependant se poursuivre pendant toute la vie de l'individu. Selon les différentes études, le torus palatin s'exprimerait environ deux fois plus fréquemment chez les femmes que chez les hommes [14,18,19,20], les types fusi- formes et plats étant les plus fréquents $[8,11,17]$. L'étiologie du torus palatin n'est pas encore clairement définie. De nombreuses théories ont été avancées pour expliquer la formation et le développement des torus. Bon nombre d'entre elles, associant les torus à l'extension d'une pathologie générale, sont abandonnées, laissant place à des théories mettant en jeu des facteurs environnementaux. En effet, les théories génétiques [20], mécanistes [21] et environnementales (climat, alimentation riche en vitamine $D$ et acides gras gamma-3 polyinsaturés) [14,22] sont aujourd'hui privilégiées par les auteurs. Il est maintenant bien établi que l'apparition du torus palatin résulte d'une action combinée de facteurs génétiques et environnementaux [19,18,22]. Selon cette théorie, le franchissement d'un seuil se révèle nécessaire pour que ces deux facteurs conduisent à la formation d'un torus [18].

Déterminer l'incidence au sein des différentes populations et entre les populations de cette exostose palatine a été l'objectif de la plupart des auteurs, d'où le très grand nombre d'études épidémiologiques sur le sujet. Kupffer (1879) [4] peut être considéré comme le premier auteur à réaliser une étude épidémiologique sur le torus palatin. Son étude se limite à une seule ethnie, ce qui conduit à affirmer que la présence de cette exostose est une caractéristique des PrussiensLithuaniens. La présence du torus palatin est par la suite relevée dans d'autres populations telles les Aïnous (Kopernicki en 1882 et Tarenetzki en 1890) [4], les Lapons (Waldeyer, 1892) [4] et les Esquimaux (Hooton, 1918) [21] amenant également ces derniers à penser que le torus est une variation anatomique caractérisant le groupe ethnique étudié. Les calculs de fréquence des torus peuvent être réalisés aussi bien sur le vivant que sur l'os sec. Ce second groupe d'étude donne une prévalence supérieure au premier. Woo [6] attribue ces différences à l'épaisseur de la muqueuse de recouvrement qui tend à dissimuler les plus petits torus. C'est pourquoi, il est préférable de traiter séparément les données obtenues sur le vivant et sur le crâne sec.

Les résultats de 120 ans de recherches épidémiologiques sur matériel sec, donnés par ordre chronologique, sont résumés dans le Tab. 1. 


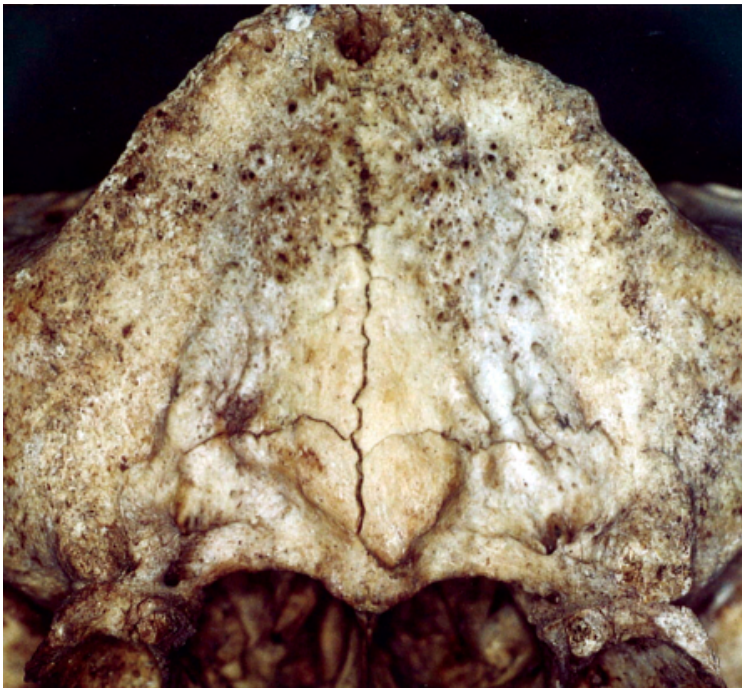

A

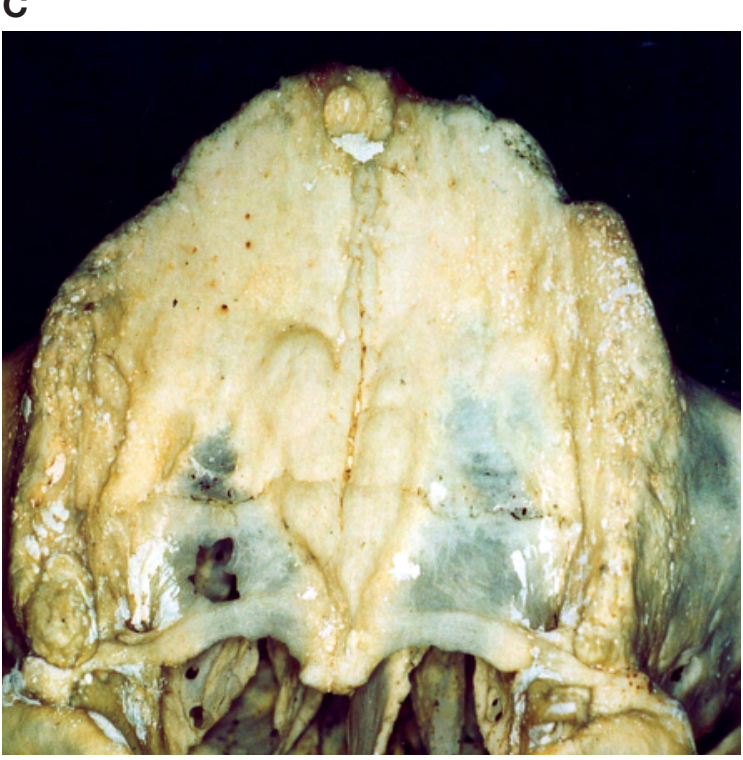

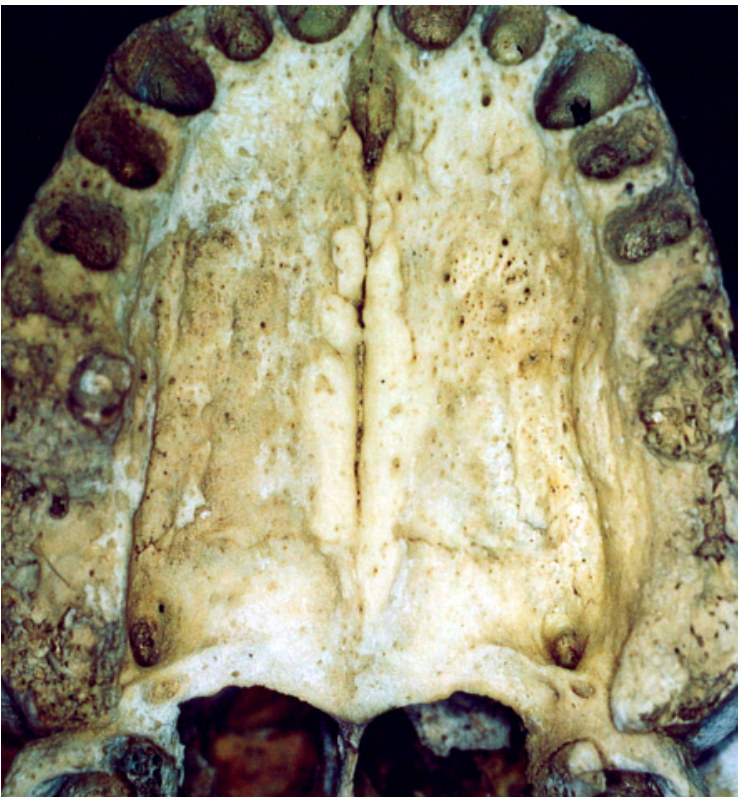

B

\section{D}

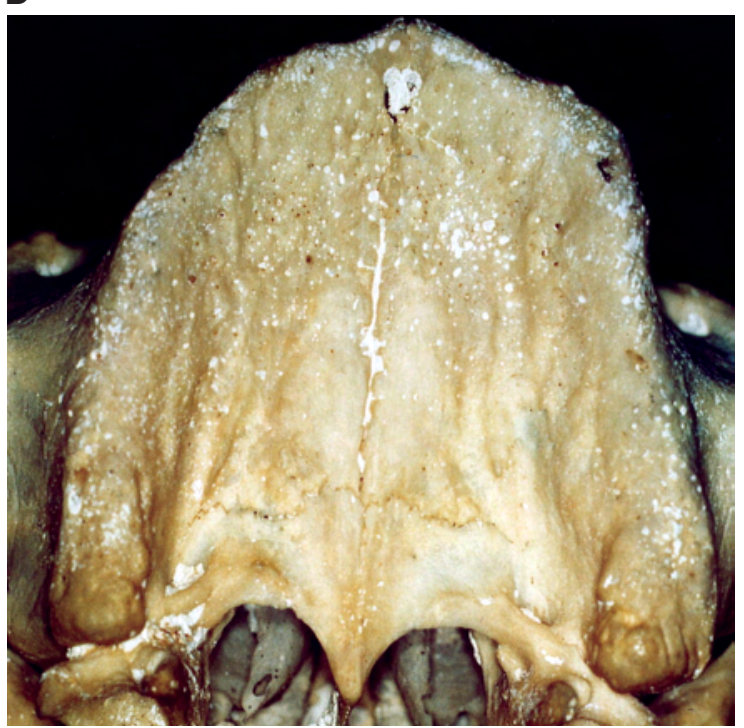

médecine

buccale

chirurgie

buccale

VOL. $11, \mathrm{~N}^{\circ} 4$ 2005

page 207

Figure 1 : Les quatre types de torus palatin d'après la classification de Thoma :

A : Torus palatin plat (ossuaire de Dabo, Bas-Rhin, 1892)

$B$ : Torus fusiforme (sans identification)

C : Torus palatin lobulaire (Alsacienne de 67 ans, 1898)

D : Torus palatin nodulaire (Alsacienne de 80 ans, 1903)

(Institut d'Anatomie Normale de Strasbourg)

The four forms of torus palatinus according to Thoma's classification :

A : Flat torus palatinus (ossuary of Dabo, Bas-Rhin, 1892)

$B$ : Spindle torus palatinus (not identified)

C. Lobular torus palatinus (Alsatian, age 67,1898 )

$D$ : Nodular torus palatinus (Alsatian, age 80,1903 )

(Normal anatomy institute of Strasbourg) 
Tableau 1: Fréquence en \% des torus palatins sur crânes secs: revue de la littérature $\mathrm{n}=$ nombre de crânes utilisés pour chaque étude

- = répartition homme/femme non précisée

Occurrence of torus palatinus on human skulls: revue of the literature.

$N=$ number of skulls examined in each study

- = Distribution men/women not specified

\begin{tabular}{|c|c|c|c|c|c|}
\hline Année & Auteurs & Population étudiée & Hommes (\%) & Femmes (\%) & Total (\%) \\
\hline 1885 & Lissauer [4] & Prussiens $(\mathrm{n}=27)$ & - & - & 66,67 \\
\hline 1885 & Lissauer [4] & Slaves Polonais $(\mathrm{n}=52)$ & - & - & 48,07 \\
\hline 1890 & Tarenetzki [4] & Slaves $(n=416)$ & - & - & 1,60 \\
\hline 1891 & Stieda [4] & Prussiens $(n=504)$ & - & - & 35,11 \\
\hline 1891 & Stieda [4] & Français (n=195) & - & - & 34,87 \\
\hline 1891 & Stieda [4] & Slaves Russes $(n=117)$ & - & - & 50,42 \\
\hline 1891 & Stieda [4] & Hottentots ( $\mathrm{n}=227)$ & - & - & 18,90 \\
\hline 1891 & Stieda [4] & Amérindiens ( $n=36$ ) & - & - & 40,00 \\
\hline 1891 & Stieda [4] & Mexicains $(n=37)$ & - & - & 44,00 \\
\hline 1891 & Stieda [4] & Péruviens ( $n=229$ ) & - & - & 56,30 \\
\hline 1891 & Stieda [4] & Esquimaux $(n=30)$ & - & - & 60,00 \\
\hline 1892 & Waldeyer [11] & Lapons $(n=49)$ & - & - & 87,70 \\
\hline 1893 & Mehnert [4] & Alsaciens $(n=113)$ & - & - & 34,51 \\
\hline 1895 & Matiegka [4] & Bohémiens ( $n=359$ ) & - & - & 22,84 \\
\hline 1896 & Jurgenson [14] & Allemands $(n=37)$ & - & - & 48,65 \\
\hline 1900 & Russel [4-11] & Esquimaux $(n=43)$ & - & - & 18,60 \\
\hline 1900 & Russel [4-11] & Amérindiens $(n=701)$ & - & - & 14,40 \\
\hline 1900 & Russel [4-11] & Péruviens $(n=436)$ & - & - & 0,20 \\
\hline 1906 & Ledouble [4] & Tourangeaux $(n=400)$ & - & - & 32,55 \\
\hline 1906 & Ledouble [4] & Lapons $(n=22)$ & - & - & 72,70 \\
\hline 1909 & Godlee [4] & Péruviens ( $n=97$ ) & - & - & 08,25 \\
\hline 1909 & Godlee [4] & Aïnous $(n=7)$ & - & - & 14,29 \\
\hline 1911 & Martens [23] & Péruviens $(\mathrm{n}=27)$ & - & - & 7,41 \\
\hline 1911 & Martens [23] & Egyptiens anciens $(n=304)$ & - & - & 12,17 \\
\hline 1911 & Martens [23] & Egyptiens modernes $(n=144)$ & - & - & 16,67 \\
\hline 1911 & Martens [23] & Hongrois $(n=85)$ & - & - & 9,41 \\
\hline 1911 & Martens [23] & Hollandais $(n=226)$ & - & - & 12,39 \\
\hline 1911 & Martens [23] & Tchèques (n=108) & - & - & 12,96 \\
\hline 1911 & Martens [23] & Croates $(n=114)$ & - & - & 7,89 \\
\hline 1911 & Martens [23] & Chinois du Sud $(n=77)$ & - & - & 10,53 \\
\hline 1918 & Hooton [21] & Amérindiens $(n=60)$ & - & - & 60,00 \\
\hline 1918 & Hooton [21] & Esquimaux $(n=60)$ & - & - & 76.70 \\
\hline 1928 & Martin [21] & Australiens ( $\mathrm{n}=?)$ & - & - & 72.00 \\
\hline 1928 & Martin [11] & Sud africains $(n=?)$ & - & - & 18.90 \\
\hline 1928 & Martin [11] & Bavarois $(\mathrm{n}=$ ?) & - & - & 13.80 \\
\hline 1928 & Martin [11] & Italiens $(\mathrm{n}=?)$ & - & - & 52.00 \\
\hline 1935 & Schreiner [24] & Esquimaux $(n=308)$ & - & - & 54.30 \\
\hline 1939 & Akabori [15] & Japonais $(n=24)$ & - & - & 43.70 \\
\hline 1940 & Hrdlicka [15-25-26] & Péruviens ( $n=465$ ) & 37,00 & 24,00 & 30.50 \\
\hline 1950 & Woo [11] & Péruviens $(n=103)$ & - & - & 18.63 \\
\hline 1950 & Woo [11] & Afro-américains $(n=575)$ & - & - & 36.00 \\
\hline 1950 & Woo [127] & Indiens $(\mathrm{n}=175)$ & - & - & 54.30 \\
\hline 1966 & Vidic [28] & Yougoslaves $(n=400)$ & 42,00 & 57,50 & 45.50 \\
\hline
\end{tabular}


Tableau 1 : (suite)

\begin{tabular}{lccccc|}
\hline Année & Auteurs & Population étudiée & Hommes (\%) & Femmes (\%) & Total (\%) \\
\hline 1972 & Larato [19] & Mexicains $(n=145)$ & - & - & 30.00 \\
1974 & Berry [29] & Danois Préhistoire $(n=97)$ & - & - & 16.30 \\
1974 & Berry [29] & Danois Moyen Age $(n=50)$ & - & - & 41.30 \\
1974 & Berry [29] & Groenlandais $(n=51)$ & - & - & 47.80 \\
1974 & Berry [29] & Esquimaux $(n=82)$ & - & - & 51.40 \\
1974 & Berry [29] & Shetlands $(n=50)$ & - & - & 40.00 \\
1974 & Berry [29] & Norvègiens $(n=28)$ & - & - & 39.10 \\
1977 & Nery [26] & Asiatiques $(n=?)$ & - & - & 46.00 \\
1977 & Nery [26] & Ethnies diverses $(n=680)$ & - & - & 40.50 \\
1981 & Kronenberger [30] & Allemands & - & - & 32.70 \\
1981 & Kronenberger [30] & Indiens et Pakistanais $(n=?)$ & - & - & 3.70 \\
1985 & Axelsson [31] & Islandais $(n=763)$ & 29,60 & 37,00 & 33.26 \\
1999 & Sonnier [26] & Américains $(n=328)$ & - & - & 56.00
\end{tabular}

\section{MATÉRIEL ET MÉTHODES}

Devant la profusion des résultats des études citées précédemment, tantôt complémentaires, tantôt contradictoires, ce travail propose d'étudier de façon systématique une série de crânes d'origines variées.

Une série de 723 crânes permet d'observer des torus palatins de taille et de morphologie différentes. Grâce à la diversité ethnique de ce matériel anatomique, l'étude des résultats permet également de donner des renseignements épidémiologiques sur l'exostose.

\section{Matériel anatomique et méthode}

L'étude se base sur l'observation de 723 crânes d'adultes conservés dans la collection d'ostéologie de l'Institut d'Anatomie Normale de Strasbourg. La diversité des origines de ce matériel anatomique et le nombre important de crânes disponibles donnent à cette collection constituée entre la fin du $19^{\circ}$ et le début du $20^{\circ}$ siècle, un intérêt épidémiologique majeur. Ce matériel provient de sources locales connues (legs de corps à l'Institut) avec certaines indications comme l'année de décès, l'âge, le sexe et l'origine du sujet, de fouilles archéologiques locales ou étrangères (Pérou, Egypte...) ainsi que du matériel anatomique de diverses provenances (Hollande, Afrique, Maghreb, Asie, Océanie...). Les crânes présentant un palais partiellement endommagé ont été exclus de l'étude.
D'autre part, plus de deux cents crânes de la collection ne portent aucune identification : ils présentent donc un intérêt épidémiologique moindre. Une partie de la collection est constituée par les crânes de 72 alsaciens collectés entre 1885 et 1908 dont l'âge et le sexe sont connus. Cette série de crânes se révèle être très intéressante, par la mise à disposition d'un échantillon de sujets contemporains d'une population géographiquement restreinte et considérée comme génétiquement homogène.

La partie postérieure du palais osseux, représentée par les lames horizontales des os palatins, présente deux dépressions de part et d'autre de la suture médiane, de profondeur variable, dont les limites sont nettes. Lorsque ces deux dépressions sont bien marquées et se rejoignent sur la ligne médiane, elles donnent à l'extrémité de la crête médiane un aspect saillant simulant un torus (Fig. 2) [23].

De même, les foramens grands palatins peuvent se présenter comme de larges et profonds sillons. La partie postérieure du palais prend ainsi une forme convexe donnant également l'illusion d'un torus (Fig. 3) [23].

\section{Mesures}

La longueur et la largeur des torus palatins sont mesurées à l'aide d'un compas et d'une règle graduée, la hauteur appréciée approximativement $(<2 \mathrm{~mm}, 2$ à $4 \mathrm{~mm},>4 \mathrm{~mm}$ ) comme le proposait Woo en 1950 [11]. En effet, le relevé exact médecine buccale chirurgie buccale

VOL. $11, N^{\circ} 4$ 2005

page 209 
médecine

buccale

chirurgie

buccale

VOL. $11, \mathrm{~N}^{\circ} 4$ 2005

page 210

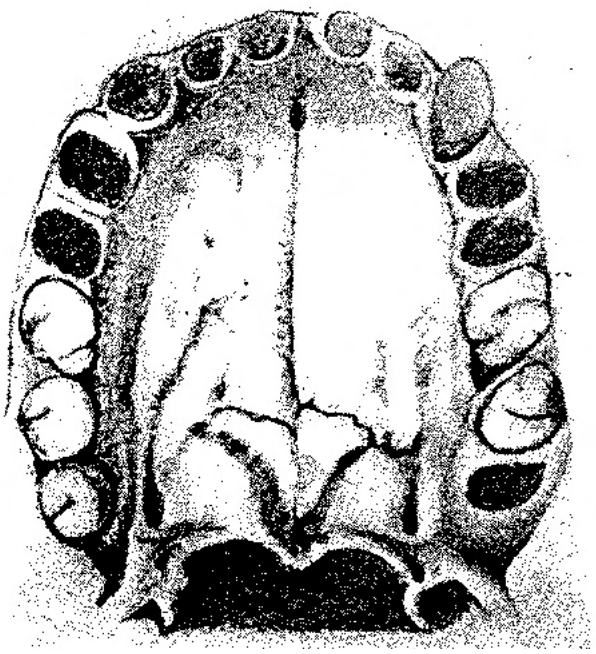

A

Figure 2 : Faux torus dû à une concavité plus marquée des zones sous-jacentes au tissu glandulaire

A : Schéma des deux dépressions osseuses se rejoignant sur la ligne médiane, simulant la présence d'un torus B : Illustration sur crâne sec (Institut d'Anatomie Normale de Strasbourg)

Not a true torus palatinus simulated by well marked hollows for adenoid tissue.

A : Not a true torus palatinus, but very marked hollows for adenoïd tissue and marked prominence between them. B : Illustration on a skull (Normal Anatomy Institute of Strasbourg)

A
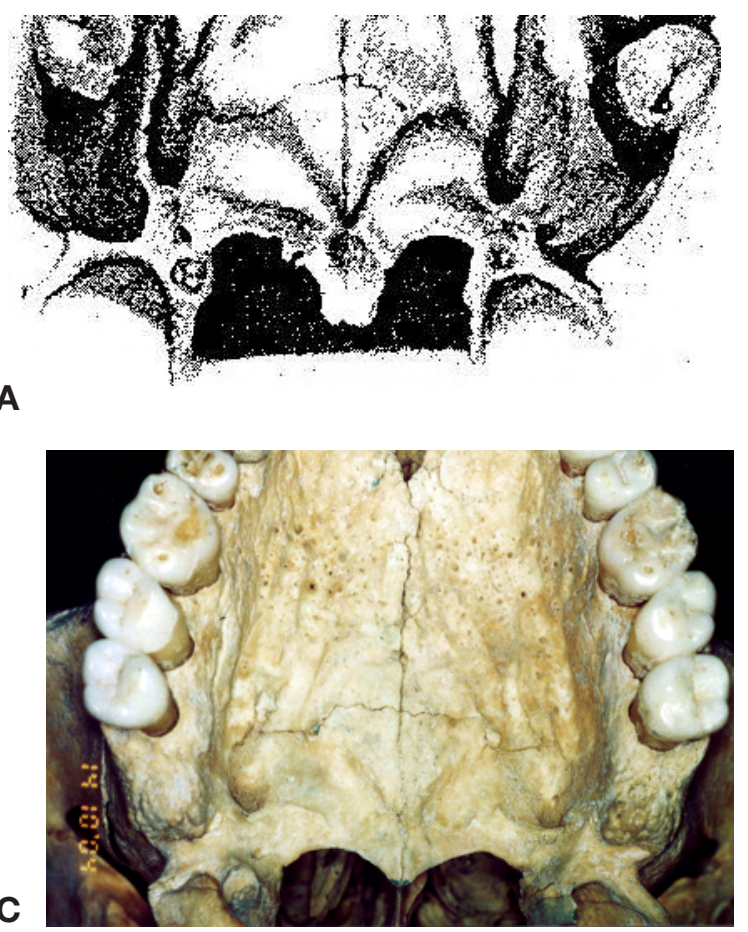

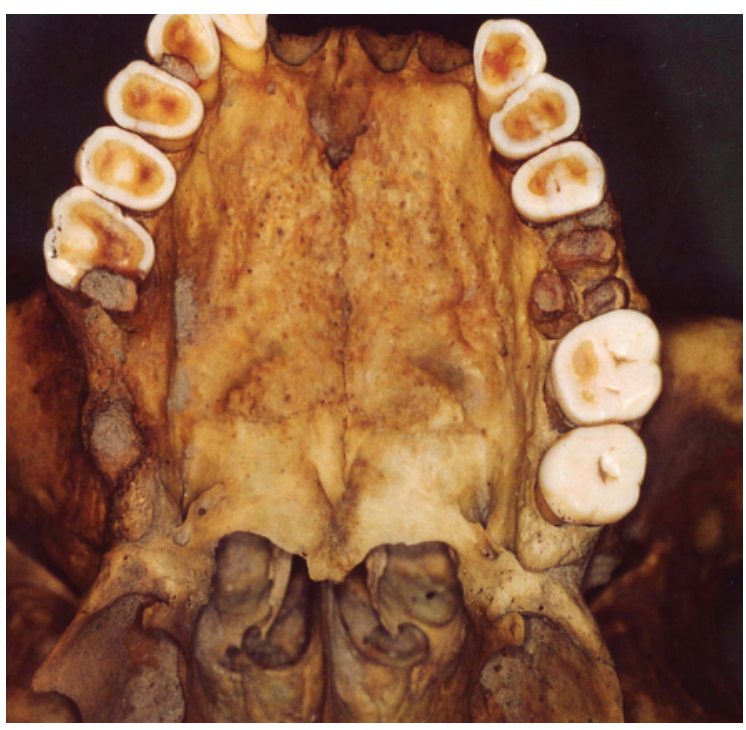

B

Figure 3 : Faux torus palatin résultant de la présence de larges foramens grands palatins : A : schéma des foramens grands palatins sous la forme de larges et profonds sillons, créant l'illusion d'un torus

B et C : Illustrations sur crânes secs (Institut d'Anatomie Normale de Strasbourg)

Not a true torus palatinus simulated by very deep vascular groove.

A : Very deep vascular groove, sharp ridge between glandular fossae, very wide palate, processes of palate bones. $B$ and $C$ : Illustrations on skulls (Normal Anatomy Institute of Strasbourg) 
de la hauteur des torus est fastidieuse et n'apporte aucune information supplémentaire à l'étude dont il est question. Les torus sont classés en petits, moyens et grands, conformément au Tab. 2.

\section{RÉSULTATS}

Sur les 723 crânes étudiés, 76 présentent un torus palatin, soit $10,5 \%$, avec la répartition suivante : $21 \%$ de petits torus, $57 \%$ de torus de taille moyenne, et $22 \%$ de grands torus.
Le Tab. 3 expose le détail de l'ensemble des observations sur crânes secs et précise le pourcentage de torus palatins observé selon la population étudiée.

Parallèlement, la Fig. 4 permet de visualiser les différences de fréquence de l'exostose selon la population étudiée.

Ces torus peuvent être classés selon les quatre types de la classification de Thoma (Fig. 1). Une nette majorité de torus plats et fusiformes (60 sur 76 soit $78 \%$ ) est également à noter. L'examen des crânes permet d'observer la présence de torus palatins chez 5 enfants (Fig. 5) et un nouveau-né.

Tableau 2 : Classement du torus palatin en fonction de sa taille, résultats obtenus suite à l'observation de 723 crânes Classification of torus palatinus according to his size. Data collected from 723 skulls.

$\begin{array}{rcccc} & & \text { Longueur }(\mathrm{mm}) & \text { Largueur }(\mathrm{mm}) & \text { Hauteur }(\mathrm{mm}) \\ \text { PORUS } & \text { Petit } & <15 & <10 & <2 \\ & \text { Moyen } & 15-25 & 10-15 & 2 \text { à } 4 \\ & \text { Grand } & >25 & >15 & >4\end{array}$

médecine

buccale

chirurgie

buccale

VOL. $11, \mathrm{~N}^{\circ} 4$ 2005

page 211

Tableau 3 : Fréquence du torus palatin, résultats obtenus suite à l'observation de 723 crânes Occurrence of torus palatinus. Data collected from 723 skulls.

\begin{tabular}{|l|c|c|c|}
\hline & Nombre & Torus palatin & Pourcentage \\
\hline Alsaciens (1885-1908) & 72 & 25 & 34,70 \\
\hline Lorrains (1885-1908) & 16 & 1 & 6,25 \\
\hline Badois (1885-1908) & 12 & 2 & 16,70 \\
\hline Suisses (Poschiavo) & 7 & 1 & 14,30 \\
\hline Hollandais (Leeuwarden) & 26 & 1 & 03,80 \\
\hline Fouilles (Alsace : Thumenau, Lupstein, & & 15 & 30,00 \\
\hline Dambach, Saverne, Mulhouse) & 50 & 1 & 01,90 \\
\hline Camerounais & 52 & 1 & 03,10 \\
\hline Tanzaniens (Dshagga) & 32 & & 1 \\
\hline Péruviens anciens & & 7 & 10,80 \\
\hline (Anca, Arica, Pachacamac) & 67 & 6 & 50,00 \\
\hline Esquimaux (Inuits) & 12 & 0 & 0 \\
\hline Tunisiens & 48 & 0 & 0 \\
\hline Egyptiens anciens & 15 & 16 & 6,00 \\
\hline Non identifiés & 265 & & \\
\hline
\end{tabular}




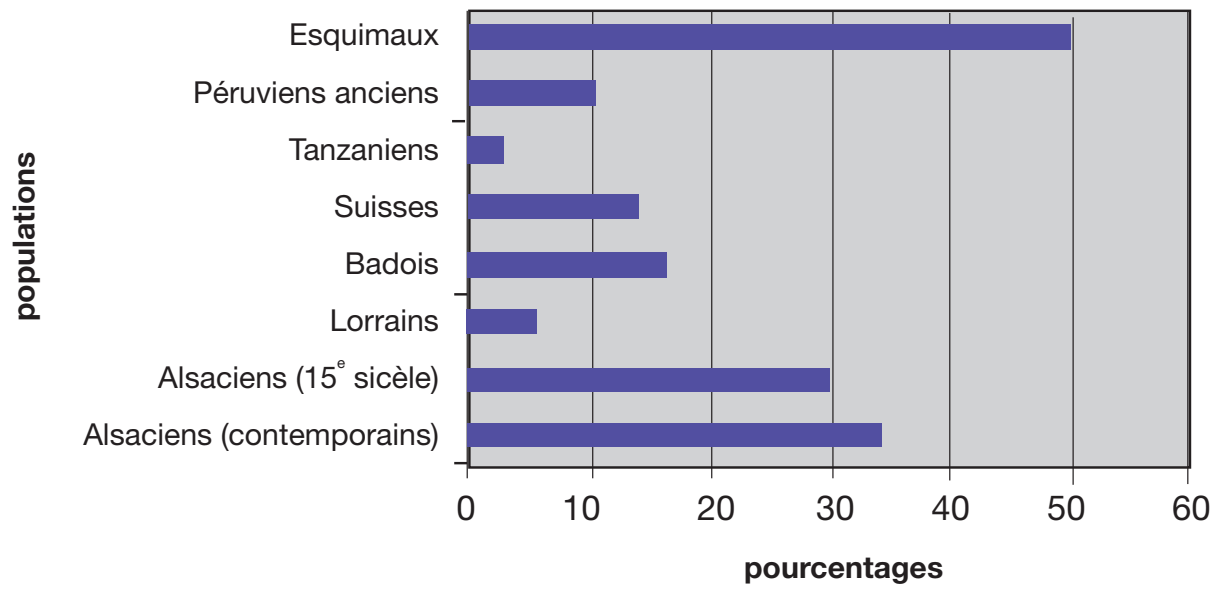

Figure 4 : Graphique des résultats les plus significatifs du Tab. 1 Diagram of the more significant results from table 1.

médecine buccale chirurgie buccale

VOL. $11, \mathrm{~N}^{\circ} 4$ 2005

page 212

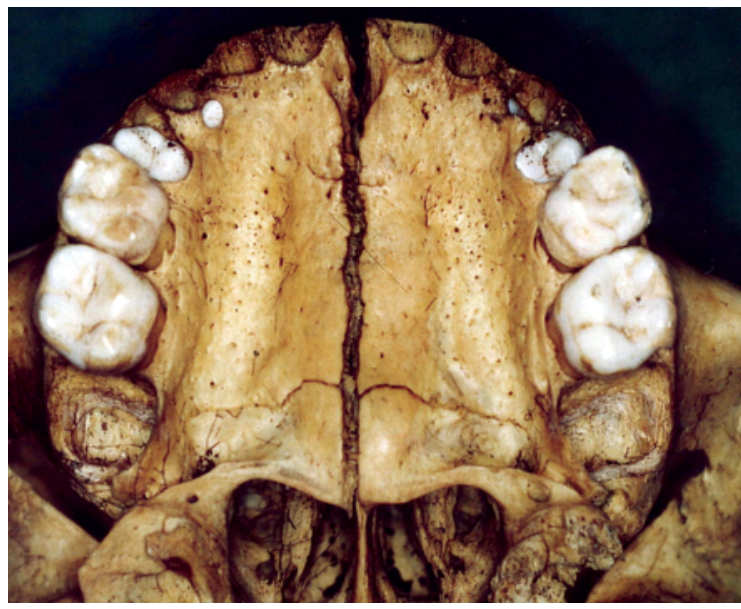

Figure 5 : Crâne d'enfant présentant un torus palatin fusiforme (Lappon de 10 ans, 19e siècle) (Institut d'Anatomie Normale de Strasbourg) Spindle torus palatinus. (Lapp, age 10, 19th century) (Normal Anatomy Institute of Strasbourg)

\section{DISCUSSION}

Il paraît important de noter la profusion d'études dans les pays nordiques et les populations asiatiques, alors que les études européennes font presque figure d'exception. Cette lacune a en partie motivé l'étude exposée ici.

La fréquence des torus palatins dans les diverses populations est similaire à celle de la littérature (Tab. 1). Malgré la rareté des crânes provenant de populations Inuits, l'étude permet d'y dénombrer une fréquence importante de torus palatins (50 \%). Il est également intéressant de relever la fréquence importante de cette exostose dans les populations rhénanes (de 14,3 à 34,7 \%).

Dans cette étude, les torus palatins plats et fusiformes constituent les types les plus fréquents (78\%). Ensemble ces deux types de torus représenteraient même $84 \%$ du total des torus observés selon Kolas [15] et $98 \%$ selon Eggen [14].

Les résultats de ces études épidémiologiques, réalisées pour certaines depuis plus d'un siècle, révèlent de grandes disparités selon les populations et les auteurs. Lors des premières études, I'utilisation combinée de matériel anthropologique et de sujets volontaires a introduit des biais modifiant la fréquence du torus palatin : les résultats recueillis à partir des crânes secs étant majorés par rapport à ceux obtenus sur le vivant. L'interprétation des résultats des études antérieures à la première moitié du $20^{\circ}$ siècle est sujette à caution. II est compréhensible que des variations existent entre des populations d'origine ethniques différentes. Cependant, l'existence de ces différences au sein même d'une population est surprenante. La présence éventuelle de facteurs étiologiques spécifiques ne peut expliquer à elle seule tous ces écarts. A partir de 1950, les études sont basées sur la classification de Thoma et séparent systématiquement les résultats obtenus sur le vivant de ceux obtenus à partir de crânes secs.

Dans leur traité de pathologies buccales et maxillo-faciales, Piette et Reychler [32] réalisent 
une synthèse de ces résultats. Pour eux, le torus palatin présente une prévalence de 1 à $7 \%$ dans les populations de type caucasien contre 35 à $80 \%$ pour les populations inuites.
Aucune méthode d'évaluation standard n'est établie jusqu'à présent. La création d'un questionnaire épidémiologique type afin de pouvoir exploiter des données internationales avec un maximum de fiabilité s'avère nécessaire.

\section{RÉFÉRENCES}

1 - Van Den Broeck AJP. On exostoses in the human skull. Acta Neerlandica Morphological Normalis et Pathological $1945 ; 5$ : 95-118.

2 - DoRRANCE GM. Torus palatinus. Dental Cosmos 1929 ; $71: 275-85$

3 - LASKER GW. Torus palatinus (an historical note). Dental Records $1952 ; 72$ : 269-73.

4 - Ledouble A. Traité des variations des os de la face ; Vigot Frères, Paris, 1906 ; pp 276-85.

5 - SHAFER J, HINE P, LEVY S. A textbook of oral pathology (3rd ed). W.B. Saunders Company Philadelphia, 1980 ; pp. 154-56.

6 - NossintCHOUc RM. Manuel d'odontologie médico-légale. Masson, Paris, 1980 ; pp. 49-51.

7 - TномA K. Torus palatinus. Int J Orthodont Oral Surg $1937 ; 23$ : 194-202.

8 - King DR, Moore GE. The prevalence of torus palatinus. J Oral Med $1971 ; 36: 113-5$.

9 - ThOMA OP, Goldman HB. Thoma's oral pathologie. CV Mosby Co, St Louis, 1960 ; 1238-47.

10 - SCHMITTBUHL M, LE MINOR JM, BOURJAT P. Hypertrophic torus palatinus simulating a palatal tumour. Rofo Fortschr Geb Rontgenstr Neuen Bildgeb Verfahr 1996 ; $2: 96-7$.

11 - Woo JK. Torus palatinus. Am J Phys Anthrop 1950 ; 8 : 80-100.

12 - KÖRNER O. Uber den Torus Palatinus. W Med 1924 ; $71: 1776-7$.

13 - KÖRnER O. Der Torus Palatinus. Zeitschrift für Ohrenheilkunde $1910 ; 61: 24-27$.

14 - EgGEN S, NATVIG B. Variation in torus palatinus prevalence in Norway. Scand J Dent Res 1994 ; 102 : 54-9.

15 - KOLAS S. The occurrence of torus palatinus and torus mandibularis in 2478 dental patients. Oral Surg 1953 ; $6: 1134-53$.

16 - ARCHER WH. Oral and maxillofacial surgery (5th ed). WB Saunders, Philadelphia, London, Toronto, 1975 ; pp. 196-204.

17 - SEAH YH. Torus palatinus and torus mandibularis: A review of the literature. Aust Dent J 1995 ; 40 : 318-21.
18 - HAUGEN LK. Palatine and mandibular tori: a morphologic study in the current Norvegian population. Acta Odontol Scand $1992 ; 50: 1-6$.

19 - Halffman CM, Scott GR, Pedersen PO. Palatine torus in the Greenlandic Norse. Am J Phys Anthropol 1992 ; $88: 145-61$.

20 - Alvesalo L, Mayhall JT, VarRela J. Torus mandibularis in 45, $X$ females (Turner syndrome). Am J Phys Anthropol $1996 ; 101: 145-9$.

21 - HоOTON EA. On certain Eskimoid characters in Icelandic skulls. Am J Phys Anthropol 1918 ; 1 : 53-76.

22 - EgGEN S, NATVIG B. Variation in torus mandibularis prevalence in Norway. Corn Dent Oral Epid 1991 ; 19 : 325.

23 - GodleE RJ. The torus palatinus. Proc R Soc Med 1909 ; $2: 175-96$.

24 - SCHREINER KE. Zur Osteologie der Lappen. $H$. Aschehoug und Co, W. Nygaard, Oslo, $1935 ; 173$.

25 - HRDLICKA A. Mandibular and maxillary exostoses. Am J Phys Anthropol $1940 ; 27: 1-67$.

26 - SONNIER K, HOMing G, COHEn M. Palatal tubercles, palatal tori and mandibular tori: prevalence and anatomical features in a U.S. population. J Periodontol 1999 ; 70 : 329-36.

27 - BERNARDA JM. Morphology and incidence of torus palatinus and mandibularis in Brazilian Indians. J Dent Res $1977 ; 56$ : 499-501.

28 - VIDIC B. Incidence of torus palatinus in Yougoslav skulls. J Dent Res 1966 ; 45 : 1511-5.

29 - GUIOT JF. Torus palatin et torus mandibulaire. Thèse Chir Dent, Strasbourg, 2002 ; 02.

30 - Kronenberger H. The torus palatinus. Anthropol Anz $1981 ; 39: 150-7$.

31 - AxELSSON G, HedegaARd B. Torus palatinus in Icelandic schoolchildren. Am J Phys Anthropol 1985 ; 67 : 105 12.

32 - Piette p, Reychler A. Traité de pathologie buccale et maxillo-faciale. De Boek Université, Bruxelles, 1991 ; pp. 1027-8.

\section{médecine buccale chirurgie buccale}

VOL. $11, \mathrm{~N}^{\circ} 4$ 2005

page 213 


\section{ERRATUM}

Une erreur de présentation s'est produite dans la mise en page de l'article: "Avulsions dentaires et kystectomies chez les patients présentant un déficit constitutionnel en facteurs de l'hémostase: conduite à tenir " Med Buccale Chir Buccale 2005; 11 (3): 121-43.

Contrairement à ce que la présentation laisse croire, il s'agit d'un article pédagogique, comme indiqué dans le sommaire page 121 et non de recommandations émanant de la Société Francophone de Médecine Buccale et de Chirurgie Buccale.

Cet article est publié sous la responsabilité de ses auteurs: Alp Alantar, Danielle Ginisty, Michel Hassin, Antoine Lafuma, Guy Princ et Natalie Stieltjes.

Le comité de rédaction présente ses excuses aux auteurs et aux lecteurs de MBCB pour cette malencontreuse confusion. 